\title{
Optimal Non-life Reinsurance under Solvency II Regime
}

\author{
Alexandru V. Asimit ${ }^{1}$ \\ Cass Business School, City University, London EC1Y 8TZ, \\ United Kingdom. E-mail: asimit@city.ac.uk \\ YiCHUN CHI \\ China Institute for Actuarial Science, Central University of Finance and Economics, Beijing, 100081, \\ China. E-mail: yichun_1982@hotmail.com \\ JUNLEI Hu \\ Cass Business School, City University, London EC1Y 8TZ, \\ United Kingdom. E-mail: Junlei.Hu.1@city.ac.uk
}

October 2, 2015

\begin{abstract}
The optimal reinsurance contract is investigated from the perspective of an insurer who would like to minimise its risk exposure under Solvency II. Under this regulatory framework, the insurer is exposed to the retained risk, reinsurance premium and change in the risk margin requirement as a result of reinsurance. Depending on how the risk margin corresponding to the reserve risk is calculated, two optimal reinsurance problems are formulated. We show that the optimal reinsurance policy can be in the form of two layers. Further, numerical examples illustrate that the optimal two-layer reinsurance contracts are only slightly different under these two methodologies.
\end{abstract}

Keywords and phrases: Optimal Reinsurance; Risk Margin; General Premium Principle; Solvency II; Technical Provision.

\section{INTRODUCTION}

A standard reinsurance contract is usually reached between two parties: the insurer, cedent, insurance buyer, or even simpler, buyer, who has an interest in transferring part of its risk to the reinsurer, also known as insurance seller, or even simpler, seller. Mathematically, let $X \geq 0$ be the total risk that the insurer faces during a fixed period, with distribution function denoted by $F(\cdot)$ and survival function $\bar{F}(\cdot)=1-F(\cdot)$. Moreover, the right end-point of $F$ is denoted by $x_{F}:=\inf \{z \in \Re: F(z)=1\}$, where $\inf \phi=+\infty$ by convention. The reinsurance seller agrees to pay, $R[X]$, the amount by which the entire loss exceeds the insurer's amount, $I[X]$, and therefore $I[X]+R[X]=X$. Two most common reinsurance contracts are the Quota-share and Stop-loss, where $I[X]=c X$ (with $0 \leq c \leq 1$ ) and

\footnotetext{
${ }^{1}$ Corresponding author. Phone: +44(0)2070405282. Fax: +44(0)2070408572.
} 
$I[X]=X \wedge M:=\min \{X, M\}$ (with $0 \leq M \leq x_{F}$ ), respectively. In order to avoid potential moral hazard issues arising from the reinsurance arrangement, the set of feasible contracts is usually given by

$$
\mathcal{F}:=\{0 \leq R[x] \leq x: R[x] \text { and } x-R[x] \text { are non-decreasing functions }\}
$$

There is a vast academic literature on identifying the optimal risk transfer for an insurance company within a one-period setting. The first attempts are attributed to Borch (1960) and Arrow (1963) who consider to minimise the variance of the insurer's retained risk and maximise the expected utility of the insurer's final wealth, respectively. Further extensions have been developed for various decision criteria (see for example, Van Heerwaarden et al., 1989, Young, 1999, Kaluska, 2001, 2005, Verlaak and Beirlant, 2003, Kaluszka and Okolewski, 2008, Ludkovski and Young, 2009 etc). Two risk measures commonly used in practice, Value-at-risk (VaR) and Conditional Value-at-risk (CVaR), are considered by Cai et al. (2008), Cheung (2010), Chi and Tan (2011) and Lu et al. (2014). The classical risk model setting has been successfully studied in the literature by Centeno and Guerra (2010) and Guerra and Centeno (2008, 2010), where a natural choice for optimisation is the maximisation of the adjustment coefficient. An optimal reinsurance contract where the counterparty default risk is incorporated into the model has been investigated by Biffis and Millossovich (2012), Bernard and Ludkovski (2012) and Asimit et al. (2013). Other extensions and variations of optimal reinsurance design studied in the literature include, for example, the model with multiple reinsurers by Chi and Meng (2014) and the model with risk margins determined via expectile risk measure by Cai and Weng (2015). A recent paper of Tan and Weng (2014) investigates the optimal reinsurance design using an empirical approach.

A relatively recent project, namely Solvency II, has been developing in order to harmonise the regulatory environment within the European Union (EU) insurance industry. This unified methodology applies to all insurance/reinsurance companies that operate in the EU insurance market and its legal framework is specified in European Commission (2009). The actual implementation of Solvency II is expected to be put in place 2016, and in the meantime, various Quantitative Impact Studies (QIS) have been performed. The main purpose of these QIS's has been to collect feedback from many insurance and reinsurance companies related to the constantly augmented Solvency II specifications. The most recent one, also known as QIS 5 (see European Commission, 2010), defines the most probable recommendations that will later lead to the implementation of Solvency II. Thus, it is very interesting to investigate the optimal reinsurance policy for an insurance company under the Solvency II Regime, which is the aim of the paper.

In this paper, we only deal with a non-life insurer, since the life reinsurance contracts have different characteristics. In addition, the buyer is regulated with the help of the Solvency II current recommendations. The aim of this paper is to identify the optimal reinsurance arrangement where the buyer's risk is quantified by the VaR plus the change in the risk margin requirement as a result of reinsurance. Traditionally, the cost of reinsurance has been considered to be solely the reinsurance premium. It would be more realistic to also include the change in the cost of capital as a result of reinsurance, which is given by the technical provisions (TP's) under the Solvency II jurisdiction. More specifically, the TP's are composed of best estimate (BE) and risk margin (RM), but only the RM component is incorporated in our optimisation objective as the BE's are calculated gross of reinsurance. Moreover, the RM's can 
be calculated via the existing proxy model in QIS5 with Log-Normal approximation or by taking into account the risk profile of the underwriting risk. Depending on how the RM corresponding to the reserve risk is calculated, two optimal reinsurance models are formulated. We show that any admissible reinsurance policy defined in (1.1) is dominated by a two-layer reinsurance contract. Further, it is very interesting to point out that our numerical analysis shows similar optimal reinsurance contracts under the two different calculation methodologies of the RM's. The rest of the paper is organised as follows: Section 2 defines our model, while Section 3 provides us with the main results. Finally, Section 4 gives some numerical examples.

\section{Model Formulation}

This section provides the details of optimal reinsurance models that are investigated in the current paper. Let us denote $P$ and $\pi(R[X])$ as the gross premium charged by the buyer and the seller, respectively, where $\pi(\cdot)$ represents the premium principle chosen by the reinsurer. To make our results accessible to many reinsurance premium principles, we follow the assumption of Chi $(2012)$, that $\pi(\cdot)$ preserves the convex order, i.e. $\pi(X) \leq \pi(Y)$ if $X \leq_{c x} Y$ which is equivalent to

$$
E X=E Y \text { and } E(X-d)_{+} \leq E(Y-d)_{+}, \text {for all } d \in \Re,
$$

where $(x)_{+}:=\max \{0, x\}$. The buyer's profit is the difference between $P-\pi(R[X])-I[X]$ and the cost of setting the TP's as defined in Solvency II. The latter is defined and detailed in European Commission (2009, 2010), but a succinct description can be found in Asimit et al. (2015), which is further used as a baseline reference in our commentary on TP's. As discussed in Section 1, for each line of business, each TP consists of BE of the liabilities and its RM, where the BE is defined as an expectation of future liabilities that are evaluated gross of any risk transfer. Thus, our optimisation objective will not take this component into account. The RM's are usually evaluated by considering four sources of risk: underwriting $(U w R)$, unavoidable market (UMR), counterparty default (CDR) and operational (OpR).

Firstly, the OpR (see for example, Asimit et al., 2015) is independent of any risk transfer, reinsurance transaction or coinsurance, and thus, we can remove this component from our analysis. In addition, the $\mathrm{UwR}$ includes the premium $(P R)$ and reserve $(R R)$ risks and its corresponding $\mathrm{RM}$ is calculated as follows

$$
R M_{U w R}(R[X]):=g\left(a_{1}(P-\pi(R[X])), b_{1} E I[X]\right),
$$

where $g(x, y):=\sqrt{x^{2}+y^{2}+x y}$ and parameters $a_{1}$ and $b_{1}$ are given by

$$
a_{1}=\lambda\left(\frac{\exp \left\{\Phi^{-1}(p) \sqrt{\log \left(1+\sigma_{P R}^{2}\right)}\right\}}{\sqrt{1+\sigma_{P R}^{2}}}-1\right), b_{1}=\lambda\left(\frac{\exp \left\{\Phi^{-1}(p) \sqrt{\log \left(1+\sigma_{R R}^{2}\right)}\right\}}{\sqrt{1+\sigma_{R R}^{2}}}-1\right) .
$$

Here, $p=99.5 \%, \Phi^{-1}(\cdot)$ is the quantile function of the standard Normal distribution and $\lambda$ represents the adjusted Cost-of-Capital rate. The parameters $\sigma_{P R}$ and $\sigma_{R R}$ are the coefficients of variation for PR and RR, respectively, as calibrated in QIS5 under the Log-Normal assumption.

Secondly, the RM for the UMR component is evaluated via the following formula

$$
R M_{U M R}(R[X]):=c E I[X] \text {, with } c=C o C \times(d-n)(d-n+1) \Delta_{n},
$$


where $C o C$ and $d$ are the Cost-of-Capital rate and modified duration of the insurer's net liability $I[X]$, respectively. In addition, $n$ is the longest duration of available risk-free financial instruments to cover $I[X]$. Moreover, $\Delta_{n}$ represents the absolute decrease of the risk-free interest for maturity $n$ under the downward stress scenario of the interest rate risk sub-module (as defined in the SCR section of European Commission, 2009).

Thirdly, the RM for CDR is given by

$$
R M_{C D R}(R[X]):=g\left(a_{2} \pi(R[X]), b_{2} E R[X]\right)
$$

with parameters $a_{2}$ and $b_{2}$ given by

$$
\begin{aligned}
a_{2}= & \lambda(1-\operatorname{Rec} R) l \sqrt{q(1-q)} \times \\
& \left(\frac{\Phi\left(\sqrt{\log \left(1+\sigma_{P R}^{2}\right)}-\Phi^{-1}(p)\right)}{1-p}-\frac{\exp \left\{\Phi^{-1}(p) \sqrt{\log \left(1+\sigma_{P R}^{2}\right)}\right\}}{\sqrt{1+\sigma_{P R}^{2}}}\right), \\
b_{2}= & \lambda(1-\operatorname{RecR}) l \sqrt{q(1-q)} \times \\
& \left(\frac{\Phi\left(\sqrt{\log \left(1+\sigma_{R R}^{2}\right)}-\Phi^{-1}(p)\right)}{1-p}-\frac{\exp \left\{\Phi^{-1}(p) \sqrt{\log \left(1+\sigma_{R R}^{2}\right)}\right\}}{\sqrt{1+\sigma_{R R}^{2}}}\right),
\end{aligned}
$$

where $l$ is a constant (usually, $3 \leq l \leq 5$ ), while $q$ and $R e c R$ represent the reinsurer one-year default probability and recovery rate, respectively.

To quantify the insurer's risk exposure, we also need to introduce VaR, which is a well-known risk measure in finance and insurance. The VaR of a generic loss variable $Z$ at a confidence level $a, V a R_{a}(Z)$, represents the minimum amount of capital that makes the insurance company to be solvent at least $a \%$ of the time, i.e.

$$
\operatorname{VaR}_{a}(Z):=\inf \left\{z \geq z_{0}: \operatorname{Pr}(Z \leq z) \geq a\right\}
$$

where $z_{0}:=\sup \{z \in \Re: \operatorname{Pr}(Z \leq z)=0\}$ represents the left-end point of the distribution function of $Z$. Recall that $\operatorname{VaR}_{p}(I[X])=\operatorname{VaR}_{p}(X)-\operatorname{VaR}_{p}(R[X])$ is true as a result of $R[X] \in \mathcal{F}$.

We are now able to introduce our optimisation problem. The classical approach in the existing literature, since the pioneering work of Cai and Tan (2007), has been to minimise the insurer's retained risk and reinsurance premium. In the Solvency II framework, it is translated to $\operatorname{Va} R_{p}(I[X])+\pi(R[X])$, since $\operatorname{VaR}_{p}(\cdot)$ is the standard risk measure used under this regulatory framework. In addition, it also includes the additional cost of capital as a result of buying reinsurance. That is, the difference between the RM's after and before the risk transfer is given by

$$
\begin{array}{r}
R M_{U w R}(R[X])+R M_{U M R}(R[X])+R M_{C D R}(R[X]) \\
-R M_{U w R}(X)-R M_{U M R}(X)-R M_{C D R}(X) .
\end{array}
$$

Recall that the BE's are calculated gross of reinsurance, and thus, our objective functions may not take the BE's into account. To simplify the representation, we further remove the last three terms in the above equation from our objective function, since they are not sensitive to the ceded loss function $R[X]$. 
Assuming that the buyer reinsures only one risk, the optimisation problem, formulated with LogNormal approximation, becomes:

$$
\min _{R \in \mathcal{F}} H_{1}(R[X])
$$

where

$$
\begin{aligned}
H_{1}(R[X]):= & R M_{U w R}(R[X])+R M_{U M R}(R[X])+R M_{C D R}(R[X]) \\
& +\pi(R[X])+\operatorname{VaR}_{p}(X)-\operatorname{VaR}_{p}(R[X]) \\
= & g\left(a_{1}(P-\pi(R[X])), b_{1} E I[X]\right)+g\left(a_{2} \pi(R[X]), b_{2} E R[X]\right) \\
& +c(E X-E R[X])+\pi(R[X])+\operatorname{VaR}_{p}(X)-\operatorname{VaR}_{p}(R[X]) .
\end{aligned}
$$

One may wonder why RR is calculated via a Log-Normal approximation, while the reinsurance premium calculation takes into account the risk profile of the underwriting policies. The insurer may choose the Standard Formula or adjust this formula by using its belief on the distribution of $X$, and the most advantageous one would be its choice.

In order to rewrite (2.1) in the absence of the Log-Normal approximation, we need to introduce another well-known risk measure, $C V a R$. This risk measure has various representations in the literature (see Acerbi and Tasche, 2002), and one of them is given by

$$
C \operatorname{Va} R_{a}(Z):=\frac{1}{1-a} \int_{a}^{1} \operatorname{VaR}_{s}(Z) d s=\operatorname{VaR}_{a}(Z)+\frac{1}{1-a} \mathbf{E}\left(Z-\operatorname{VaR}_{a}(Z)\right)_{+} .
$$

Interestingly, this risk measure is a special case of the Haezendonck-Goovaerts class, which was introduced many years ago by Haezendonck and Goovaerts (1982). Further details can be found in Bellini and Rosazza Gianin (2012), Goovaerts et al. (2004 and 2012) and the references therein.

Recall that all previous RM's have been calculated based on the Standard Formula from Solvency II, where the Log-Normal approximation has been used in the definition of RM's for UwR and CDR. An alternative calculation of the RR contributions is to take into account the actual variation within the risk itself, i.e. $I[X]$ and $R[X]$ for the UwR and CDR, respectively. Concretely, the Log-Normal approximation for the RM's of UwR and CDR showed that

$$
\lambda\left(\operatorname{VaR}_{p}(I[X])-E I[X]\right) \approx b_{1} E I[X]
$$

and

$$
\vartheta\left(C V a R_{p}(R[X])-\operatorname{VaR}_{p}(R[X])\right) \approx b_{2} E R[X]
$$

where $\vartheta=\lambda(1-\operatorname{Rec} R) l \sqrt{q(1-q)}$. It has been implicitly assumed in the last two equations that $I[X]$ and $R[X]$ are Log-Normal distributed with the same coefficient of variation. This standard assumption is acceptable in the Solvency II framework, and the values of $\sigma_{R R}$ for the recognised nine lines of business can be found in European Commission (2010) or Asimit et al. (2015). By the above approximations, we should guarantee that $\operatorname{VaR}_{p}(I[X]) \geq E I[X]$, and the RM's for UwR and CDR can be rewritten by

$$
R M_{U w R}(R[X])=g\left(a_{1}(P-\pi(R[X])), \lambda(\operatorname{VaR}(I[X])-E I[X])\right)
$$


and

$$
R M_{C D R}(R[X])=g\left(a_{2} \pi(R[X]), \vartheta\left(C \operatorname{VaR}_{p}(R[X])-\operatorname{VaR}_{p}(R[X])\right)\right)
$$

respectively. Thus, if we refrain ourselves from using the Log-Normal approximation in the RM calculation corresponding to RR, the optimisation problem (2.1) can be rewritten as follows:

$$
\min _{R \in \mathcal{F}} H_{2}(R[X])
$$

where

$$
\begin{aligned}
H_{2}(R[X]):= & g\left(a_{1}(P-\pi(R[X])), \lambda\left(\operatorname{VaR}_{p}(I[X])-E I[X]\right)\right)+c(E X-E R[X]) \\
& +g\left(a_{2} \pi(R[X]), \vartheta\left(C V_{a} R_{p}(R[X])-V_{a} R_{p}(R[X])\right)\right)+\pi(R[X]) \\
& +V_{a} R_{p}(X)-\operatorname{VaR}_{p}(R[X]) .
\end{aligned}
$$

\section{Optimal Reinsurance DESIGN}

In this section, we proceed with solving the optimal reinsurance problems (2.1) and (2.3). Note that $a_{1}, a_{2}, b_{1}, b_{2} \in(0,1)$, since $C o C, \lambda \in(0,1)$ and the market wide estimates for $\sigma_{P R}$ and $\sigma_{R R}$ showed to be less than $22 \%$. Therefore, it is not difficult to see that the objective function from (2.1) is increasing in $\pi(R[X])$. Consequently, the first step in solving (2.1) is to find the solutions to the following optimisation problem

$$
\begin{aligned}
& \arg \min _{R \in \mathcal{F}} \pi(R[X]), \\
& \text { subject to } E R[X]=\mu \in[0, E X], \\
& \qquad V_{a} R_{p}(R[X])=\nu, \\
& \pi(R[X]) \leq P .
\end{aligned}
$$

Clearly, the last inequality constraint ensures that the reinsurance premium could not exceed the insurer's gross premium.

Before solving the above minimisation problem, we first define a layer $(a, b]$ of a risk $Z$ by

$$
\mathcal{L}_{(a, b]}(Z):=(Z-a)_{+} \wedge(b-a)=(Z-a)_{+}-(Z-b)_{+}, 0 \leq a \leq b .
$$

Next, the analysis of the above infinite-dimensional optimisation problem can be reduced to studying a certain finite-dimensional one, as shown in the following theorem.

Theorem 3.1. For any admissible ceded loss function $R[X]$, denote $\mu=E R[X]$ and $\nu=\operatorname{Va} R_{p}(R[X])$, then there must exist an admissible ceded loss function $\tilde{R}[X]$ in the form of

$$
\tilde{R}[X]= \begin{cases}X \wedge \nu+\mathcal{L}_{\left(\operatorname{VaR}_{p}(X), t\right]}(X), & E(X \wedge \nu) \leq \mu \\ X \wedge z+\mathcal{L}_{\left(\operatorname{VaR}_{p}(X)-\nu+z, \operatorname{VaR}_{p}(X)\right]}(X), & \text { otherwise, }\end{cases}
$$

for some $z \in[0, \nu]$ and $t \geq \operatorname{VaR}_{p}(X)$ such that

$$
C \operatorname{VaR}_{p}(R[X]) \geq \operatorname{CVaR}(\tilde{R}[X]), \operatorname{VaR}_{p}(\tilde{R}[X])=\operatorname{VaR}_{p}(R[X]) \quad \text { and } \quad \tilde{R}[X] \leq_{c x} R[X] .
$$


Moreover, we have

$$
C V a R_{p}(\tilde{R}[X])-\operatorname{VaR}_{p}(\tilde{R}[X])=\frac{1}{1-p}(\mu-E(X \wedge \nu))_{+} .
$$

Proof. Using a proof similar to that of Theorem 3.2 in Chi (2012), we get that there exists an admissible ceded loss function in the form of

$$
\hat{R}[X]=\mathcal{L}_{\left(0, d_{1}\right]}(X)+\mathcal{L}_{\left(\operatorname{VaR}_{p}(X)+d_{1}-\nu, d_{2}\right]}(X),
$$

for some $d_{1} \in[0, \nu]$ and $d_{2} \in\left[\operatorname{VaR}(X), x_{F}\right]$ such that

$$
\hat{R}[X] \leq_{c x} R[X], \quad \operatorname{VaR}_{p}(\hat{R}[X])=\nu \quad \text { and } \quad C V a R_{p}(\hat{R}[X])=C V a R_{p}(R[X])
$$

Now, building upon $\hat{R}[X]$, we can construct an admissible ceded loss function $\tilde{R}[X]$ as in $(3.1)$, where $t$ and $z$ are determined by $E \tilde{R}[X]=E \hat{R}[X]=\mu$. More specifically, if $E[X \wedge \nu] \leq \mu$, it is not difficult to find that $\mathcal{H}(y):=E\left(X \wedge \nu+\mathcal{L}_{\left(\operatorname{VaR}_{p}(X), y\right]}(X)\right), y \geq \operatorname{VaR}_{p}(X)$, is a non-decreasing continuous function with

$$
\mathcal{H}\left(\operatorname{VaR}_{p}(X)\right)=E(X \wedge \nu) \leq \mu \quad \text { and } \quad \mathcal{H}\left(d_{2}\right) \geq E \hat{R}[X]=\mu
$$

Thus, there must exist a $t \in\left[\operatorname{VaR}_{p}(X), d_{2}\right]$ such that $E \tilde{R}(X)=\mathcal{H}(t)=\mu$. Now, if $E(X \wedge \nu)>\mu$, we have $E \tilde{\mathcal{H}}\left[X ; d_{1}\right] \leq E \hat{R}[X]=\mu$ and $E \tilde{\mathcal{H}}[X ; \nu]=E(X \wedge \nu)>\mu$, where

$$
\tilde{\mathcal{H}}[X ; y]:=X \wedge y+\mathcal{L}_{\left(\operatorname{VaR}_{p}(X)-\nu+y, \operatorname{VaR}_{p}(X)\right]}[X], \quad d_{1} \leq y \leq \nu .
$$

Moreover, $E \tilde{\mathcal{H}}[X ; y]$ is continuous and non-decreasing in $y$, and therefore, there must exist a $z \in\left[d_{1}, \nu\right]$ such that $E \tilde{R}[X]=E \tilde{\mathcal{H}}[X ; z]=\mu$.

Further, we can see from the definition of $\tilde{R}[X]$ that $\operatorname{VaR}_{p}(\tilde{R}[X])=\nu$ and $(3.2)$ are true. By comparing $\tilde{R}(X)$ with $\hat{R}(X)$, we note that $\hat{R}(X) \geq \tilde{R}(X)$ and $\hat{R}(X) \leq \tilde{R}(X)$ are true for any $X \geq V a R_{p}(X)$ and $0 \leq X \leq \operatorname{VaR}_{p}(X)$, respectively. In other words, $\hat{R}$ up-crosses $\tilde{R}$, and in turn, it follows from Lemma 3 of Ohlin (1969) that $\tilde{R}[X] \leq_{c x} R[X]$. It is well-known that the CVaR risk measure preserves the convex order, and thus, we have $C V a R_{p}(R[X]) \geq C V a R_{p}(\tilde{R}[X])$. The proof is now complete.

By the above theorem, we know that any admissible ceded loss function $R$ is dominated by $\tilde{R}$ (as defined in (3.1)). According to the definition of $\tilde{R}$, one can find that the parameters $z$ and $t$ when $\mu$ and $\nu$ are known. Moreover, as $\tilde{R}[X]$ is a function of $\mu$ and $\nu$, we rewrite it as $\tilde{R}[X ; \mu, \nu]$ in order to emphasise this dependence. As a result, the study of optimal reinsurance problem (2.1) can be simplified to solving a minimisation problem of two decision variables $\mu$ and $\nu$. Before solving this minimisation problem, it is necessary to elaborate the relationship between $\mu$ and $\nu$. Specifically, for any $R \in \mathcal{F}$ with $\operatorname{VaR}_{p}(R[X])=\nu$, we have

$$
\mathcal{L}_{\left(\operatorname{VaR}_{p}(X)-\nu, \operatorname{VaR}_{p}(X)\right]}(X) \leq R[X] \leq X \wedge \nu+\left(X-\operatorname{VaR}_{p}(X)\right)_{+}
$$

Consequently, $(\mu, \nu)$ should satisfy

$$
\int_{\operatorname{VaR}_{p}(X)-\nu}^{V a R_{p}(X)} \bar{F}(x) d x \leq \mu \leq E X-\int_{\nu}^{V a R_{p}(X)} \bar{F}(x) d x \quad \text { and } \quad \nu \leq \operatorname{VaR}_{p}(X) .
$$

Thus, optimisation problem (2.1) is equivalent to

$$
\min _{\substack{\left\{(\mu, \nu) \in \Re_{+}^{2}:(3.3) \text { is satisfied } \\ \pi(\tilde{R}[X ; \mu, \nu]) \leq P\right\}}} h_{1}(\mu, \nu), \text { where } h_{1}(\mu, \nu):=H_{1}(\tilde{R}[X ; \mu, \nu]) .
$$


The analysis of the above optimisation problem can be further simplified by using the following proposition.

Proposition 3.1. For any $0 \leq \mu \leq E X$, we have

$$
h_{1}(\mu, \nu) \geq h_{1}(\mu, \nu(\mu)), \forall 0 \leq \nu \leq \nu(\mu)
$$

where

$$
\nu(\mu):=\sup \left\{0 \leq t \leq \operatorname{VaR}_{p}(X): E(X \wedge t) \leq \mu\right\}
$$

Proof. For any $0 \leq \nu^{1}<\nu^{2} \leq \nu(\mu)$, we can show that

$$
\tilde{R}\left[X ; \mu, \nu^{2}\right] \leq_{c x} \tilde{R}\left[X ; \mu, \nu^{1}\right]
$$

Specifically, noting that $E\left(X \wedge \nu^{i}\right) \leq \mu$ for $i=1,2$, it can be seen from (3.1) that $\tilde{R}\left[X ; \mu, \nu^{1}\right]$ up-crosses $\tilde{R}\left[X ; \mu, \nu^{2}\right]$ and that $E \tilde{R}\left[X ; \mu, \nu^{i}\right]=\mu$ for $i=1,2$. Then, (3.6) could be obtained by using Lemma 3 from Ohlin (1969).

Further, $\pi\left(\tilde{R}\left[X ; \mu, \nu^{2}\right]\right) \leq \pi\left(\tilde{R}\left[X ; \mu, \nu^{1}\right]\right)$ follows from the assumption that $\pi($.$) preserves the convex$ order. Recall that $h_{1}(\mu, \nu)$ is non-increasing in $\nu$ and is non-decreasing in $\pi(\tilde{R}[X ; \mu, \nu])$. Hence, the final result is obtained, which concludes our proof.

We now turn to study optimal reinsurance model (2.3). Recall that $a_{1}, a_{2}, b_{1}, b_{2} \in(0,1)$. It is not difficult to find that the objective function $H_{2}(R[X])$ is increasing in $\pi(R[X])$ and $C V a R_{p}(R[X])$. Further, using Theorem 3.1, we know that any admissible ceded loss function $R[X]$ is sub-optimal to $\tilde{R}[X ; \mu, \nu]$ where $\mu=E R[X]$ and $\nu=\operatorname{VaR}_{p}(R[X])$. As a result, the analysis of optimal reinsurance model (2.3) can be simplified to solving an optimisation problem of two decision variables $\mu$ and $\nu$. However, in contrast to the optimisation problem $(3.4),(\mu, \nu)$ is subject to more constraints. Specifically, in addition to (3.3), another constraint, $\nu \leq \operatorname{VaR}_{p}(X)+\mu-E X$ (in order to guarantee the feasibility constraint $\left.\operatorname{VaR}_{p}(I[X]) \geq E I[X]\right)$, is needed as a consequence of removing the Log-Normal approximation. Thus, the optimisation problem (2.3) is equivalent to

$$
\min _{\substack{\left\{(\mu, \nu) \in \Re_{+}^{2}: \nu \leq V a R_{p}(X)+\mu-E X \\ \pi(\tilde{R}[X ; \mu, \nu]) \leq P \text { and }(3.3) \text { is satisfied }\right\}}} h_{2}(\mu, \nu), \quad \text { where } h_{2}(\mu, \nu):=H_{2}(\tilde{R}[X ; \mu, \nu]) .
$$

We try to further reduce the dimension of the above minimisation problem, and define

$$
\phi(\mu)=: \mu-E\left(X \wedge\left(\operatorname{VaR}_{p}(X)+\mu-E X\right)\right), 0 \leq \mu \leq E X .
$$

Noting that $p=99.5 \%$, we assume that $\operatorname{VaR}_{p}(X) \geq E X$ in the remainder of the paper. Thus, $\phi(\mu)$ is a non-decreasing and continuous function with $\phi(0) \leq 0$ and $\phi(E X) \geq 0$. Consequently,

$$
\mu_{0}:=\inf \{0 \leq \mu \leq E X: \phi(\mu)=0\}
$$

is well-defined.

Proposition 3.2. If $\mu \geq \mu_{0}$, we get

$$
h_{2}(\mu, \nu) \geq h_{2}\left(\mu, \operatorname{VaR}_{p}(X)+\mu-E X\right), \forall 0 \leq \nu \leq \operatorname{VaR} R_{p}(X)+\mu-E X
$$


Whenever $0 \leq \mu<\mu_{0}$, we have

$$
h_{2}(\mu, \nu) \geq h_{2}(\mu, \nu(\mu)), \forall 0 \leq \nu \leq \nu(\mu)
$$

where $\nu(\mu) \in\left[0, \operatorname{VaR}_{p}(X)\right)$ is defined in $(3.5)$.

Proof. It is first assumed that $\mu \geq \mu_{0}$. Now, $E(X \wedge \nu) \leq \mu$ holds as a result of $\nu \leq \operatorname{VaR}(X)+\mu-E X$. We can see from the proof of Proposition 3.1 that

$$
\pi\left(\tilde{R}\left[X ; \mu, \nu^{2}\right]\right) \leq \pi\left(\tilde{R}\left[X ; \mu, \nu^{1}\right]\right) \text { for any } 0 \leq \nu^{1}<\nu^{2} \leq \operatorname{VaR}(X)+\mu-E X
$$

Furthermore, relation $(3.2)$ yields that $C \operatorname{Va} R_{p}(\tilde{R}[X ; \mu, \nu])-\operatorname{Va} R_{p}(\tilde{R}[X ; \mu, \nu])$ is non-increasing in $\nu$. Thus, $h_{2}(\mu, \nu)$ is non-increasing in $\nu$ by keeping in mind that $h_{2}(\cdot)$ is non-decreasing in $\pi(\cdot)$ and $C V a R_{p}(\cdot)$. Hence, our first claim is justified in full.

The case in which $0 \leq \mu<\mu_{0}$ can be dealt as above. The proof is finally completed.

Remark 3.1. Proposition 3.2 says that whenever $\mu \geq \mu_{0}$, any admissible ceded loss function $\tilde{R}[X ; \mu, \nu]$ is dominated by $\tilde{R}\left[X ; \mu, \operatorname{VaR}_{p}(X)+\mu-E X\right]$, which is well-defined as

$$
E\left(X \wedge\left(\operatorname{VaR}_{p}(X)+\mu-E X\right)\right)+E\left(X-\operatorname{VaR}_{p}(X)\right)_{+}=E X-\int_{V a R_{p}(X)+\mu-E X}^{\operatorname{VaR}(X)} \bar{F}(t) d t \geq \mu
$$

Otherwise, for $0 \leq \mu<\mu_{0}$, Proposition 3.2 implies that the optimal $\nu$ must be attained over the interval $\left[\nu(\mu), \operatorname{VaR}_{p}(X)+\mu-E X\right] \cap \mathfrak{N}_{\mu}$, where

$$
\mathfrak{N}_{\mu}:=\left\{\nu \in\left[0, \operatorname{VaR}_{p}(X)+\mu-E X\right]: \text { (3.3) is satisfied. }\right\}, 0 \leq \mu \leq E X .
$$

Now, for any $\nu \in\left[\nu(\mu), \operatorname{VaR}_{p}(X)+\mu-E X\right] \cap \mathfrak{N}_{\mu}$, we have $E(X \wedge \nu) \geq E(X \wedge \nu(\mu))=\mu$. This, together with (3.2), yields that

$$
C \operatorname{VaR}_{p}(\tilde{R}[X ; \mu, \nu])-\operatorname{VaR}_{p}(\tilde{R}[X ; \mu, \nu])=0
$$

which in turn implies that the third term of $h_{2}(\cdot)$ from (2.3) is reduced to

$$
R M_{C D R}(\tilde{R}[X ; \mu, \nu])=a_{2} \pi(\tilde{R}[X ; \mu, \nu])
$$

Collecting the above arguments, the analysis of minimisation problem (3.7) can be reduced to solving two simpler optimisation problems:

$$
\text { (i) } \min _{\substack{\mu_{0} \leq \mu \\ \pi\left(\tilde{R}\left[X ; \mu, \operatorname{Va} R_{p}(X)+\mu-E X\right]\right) \leq P}} h_{2}\left(\mu, \operatorname{VaR}_{p}(X)+\mu-E X\right) ;
$$

where

$$
\mathcal{R}:=\left\{(\mu, \nu) \in \Re_{+}^{2}: 0 \leq \mu<\mu_{0}, \nu \in\left[\nu(\mu), \operatorname{VaR} R_{p}(X)+\mu-E X\right] \cap \mathfrak{N}_{\mu}\right\}
$$

It seems impossible to find closed-form solutions without specifying the premium principle $\pi(\cdot)$. Thus, some well-known premium principles are investigated in the next section in order to derive the optimal parameters of the two-layer reinsurance. 


\section{EXAMPLES AND NUMERICAL ANALYSIS}

The final section of the paper provides the solutions to optimal reinsurance problems (2.1) and (2.3) for some well-known premium principles. Initially, closed-form solutions are found for expected value premium principle, which is formally defined as

$$
\pi(\cdot)=(1+\rho) E[\cdot]
$$

where $\rho>0$ represents the safety loading coefficient. Numerical solutions are then investigated for a large class of premium principles, known as Wang's class, for which we need to assume that the loss sample space is composed of a finite number of possible outcomes.

4.1. Expected value premium principle. As anticipated, we now find the closed-form solutions of optimisation problems (2.1) and (2.3) under the expected value premium principle assumption. By Theorem 3.1, the optimal ceded loss function can be in the form of (3.1) and the analysis reduces to solving the minimisation problems (3.4) and (3.7). A two-step procedure is used to solve these problems, where the first step derives the optimal $\nu$ for a fixed $\mu$, while the second step provides the global optimal solution. Noting that $\mu$ is upper-bounded by $\frac{P}{1+\rho}$ since $\pi(\tilde{R}[X ; \mu, \nu]) \leq P$, the value of $P$ is critical to the set of feasible solutions. Thus, our analysis is divided accordingly. As reinsurance is expected to be more costly than insurance, the economic constraint, $P \leq(1+\rho) E X$, is made in this subsection.

We first study the optimisation problem (2.1), where Log-Normal approximation is used. By some simple algebra, the objective function $h_{1}(\cdot)$ can be rewritten as follows:

$$
h_{1}(\mu, \nu)=c E X+\left(C_{2}-c+1+\rho\right) \mu+C_{1} \sqrt{\left(\mu-\frac{B}{2 C_{1}^{2}}\right)^{2}+\frac{3 a_{1}^{2} b_{1}^{2}}{4 C_{1}^{4}}(P-(1+\rho) E X)^{2}}+V_{a} R_{p}(X)-\nu,
$$

where $C_{i}:=g\left(a_{i}(1+\rho), b_{i}\right), i=1,2$ and $B:=2 a_{1}^{2} P(1+\rho)+2 b_{1}^{2} E X+a_{1} b_{1}(P+(1+\rho) E X)$.

In the first step, note that $h_{1}(\mu, \nu)$ is non-increasing in $\nu$, and then we have $h_{1}(\mu, \nu) \geq h_{1}\left(\mu, \nu^{*}(\mu)\right)$, where $\nu^{*}(\mu)=\sup \left\{0 \leq t \leq \operatorname{VaR}_{p}(X): \int_{\operatorname{VaR}_{p}(X)-t}^{\operatorname{Va} R_{p}(X)} \bar{F}(z) d z \leq \mu\right\}$. The second step is used to solve the following optimisation problem $\min _{\mu \in[0, P /(1+\rho)]} h_{1}\left(\mu, \nu^{*}(\mu)\right)$.

1a) If $P /(1+\rho)<E\left(X \wedge \operatorname{VaR}_{p}(X)\right)$, then for any $\mu \in[0, P /(1+\rho)]$, we have $\nu^{*}(\mu)<\operatorname{VaR}(X)$, and together with $(4.2)$ we get that

$$
\begin{aligned}
h_{1}\left(\mu, \nu^{*}(\mu)\right)= & \left(C_{2}-c+1+\rho\right) \mu+C_{1} \sqrt{\left(\mu-\frac{B}{2 C_{1}^{2}}\right)^{2}+\frac{3 a_{1}^{2} b_{1}^{2}}{4 C_{1}^{4}}(P-(1+\rho) E X)^{2}} \\
& +\operatorname{VaR}_{p}(X)-\nu^{*}(\mu)+c E X .
\end{aligned}
$$

Differentiating $h_{1}\left(\mu, \nu^{*}(\mu)\right)$ with respect to $\mu$, we obtain

$$
\begin{aligned}
h_{1}^{\prime}\left(\mu, \nu^{*}(\mu)\right)= & \frac{C_{1}\left(\mu-\frac{B}{2 C_{1}^{2}}\right)}{\sqrt{\left(\mu-\frac{B}{2 C_{1}^{2}}\right)^{2}+\frac{3 a_{1}^{2} b_{1}^{2}}{4 C_{1}^{4}}(P-(1+\rho) E X)^{2}}}-\frac{1}{\bar{F}\left(\operatorname{VaR}_{p}(X)-\nu^{*}(\mu)\right)} \\
& +C_{2}-c+1+\rho .
\end{aligned}
$$

One may find that $h_{1}^{\prime}\left(\mu, \nu^{*}(\mu)\right)$ is non-decreasing. Hence, the minimal value of $h_{1}\left(\mu, \nu^{*}(\mu)\right)$ is obtained at

$$
\mu_{1}^{*}=\left(\sup \left\{0 \leq \mu \leq P /(1+\rho): h_{1}^{\prime}\left(\mu, \nu^{*}(\mu)\right) \leq 0\right\}\right)_{+}
$$


where $\sup \emptyset=-\infty$ by convention.

1b) Otherwise, if $P /(1+\rho) \geq E\left(X \wedge V a R_{p}(X)\right)$, we separate the discussion into two subcases: $\mu \in\left[0, E\left(X \wedge \operatorname{VaR}_{p}(X)\right)\right)$ and $\mu \in\left[E\left(X \wedge \operatorname{VaR}_{p}(X)\right), P /(1+\rho)\right]$.

For any $\mu \in\left[0, E\left(X \wedge \operatorname{VaR}_{p}(X)\right)\right)$, similar arguments to those used in case (1a), the minimal value of $h_{1}\left(\mu, \nu^{*}(\mu)\right)$ is attainable at

$$
\mu_{2}^{*}=\left(\sup \left\{0 \leq \mu<E\left(X \wedge \operatorname{VaR}_{p}(X)\right): h_{1}^{\prime}\left(\mu, \nu^{*}(\mu)\right) \leq 0\right\}\right)_{+},
$$

where $h_{1}^{\prime}\left(\mu, \nu^{*}(\mu)\right)$ is given in $(4.3)$.

If $\mu \in\left[E\left(X \wedge \operatorname{VaR}_{p}(X)\right), P /(1+\rho)\right]$, then we have $\nu^{*}(\mu)=\operatorname{VaR}(X)$ and

$$
h_{1}\left(\mu, \nu^{*}(\mu)\right)=c E X+\left(C_{2}-c+1+\rho\right) \mu+C_{1} \sqrt{\left(\mu-\frac{B}{2 C_{1}^{2}}\right)^{2}+\frac{3 a_{1}^{2} b_{1}^{2}}{4 C_{1}^{4}}(P-(1+\rho) E X)^{2}} .
$$

Clearly, $h_{1}\left(\mu, \nu^{*}(\mu)\right)$ is a convex function and its minimum is attained at

$$
\begin{aligned}
\mu_{3}^{*}= & \max \left\{\operatorname { s u p } \left\{E\left[X \wedge \operatorname{VaR}_{p}(X)\right] \leq \mu \leq \frac{P}{1+\rho}:\right.\right. \\
& \left.\left.C_{2}-c+1+\rho+\frac{C_{1}\left(\mu-\frac{B}{2 C_{1}^{2}}\right)}{\sqrt{\left(\mu-\frac{B}{2 C_{1}^{2}}\right)^{2}+\frac{3 a_{1}^{2} b_{1}^{2}}{4 C_{1}^{4}}(P-(1+\rho) E X)^{2}}} \leq 0\right\}, E\left(X \wedge \operatorname{VaR} R_{p}(X)\right)\right\} .
\end{aligned}
$$

Finally, the minimum between $h_{1}\left(\mu_{2}^{*}, \nu^{*}\left(\mu_{2}^{*}\right)\right)$ and $h_{1}\left(\mu_{3}^{*}, \nu^{*}\left(\mu_{3}^{*}\right)\right)$ provides the global optimal solution to $(2.1)$.

Now, we turn our attention to solving the optimisation problem (2.3), where the Log-Normal approximation is not used to estimate the $R R$ within the $U w R$ and $C D R$. It has been found at the end of Remark 3.1 that for any $\mu \geq \mu_{0}$, we only need to solve a minimisation problem in one variable, namely $\mu$. The same conclusion is drawn in the next proposition for the other case in which $0 \leq \mu<\mu_{0}$.

Proposition 4.1. Assume that the reinsurance premium is calculated by the expected value principle as given in (4.1). Whenever $0 \leq \mu<\mu_{0}$, we have $h_{2}(\mu, \nu) \geq h_{2}\left(\mu, \nu^{*}(\mu)\right)$ for all $\nu \in \mathfrak{N}_{\mu}$, where

$$
\nu^{*}(\mu):=\min \left\{\operatorname{VaR}_{p}(X)+\mu-E X, \mathfrak{M}(\mu)\right\}
$$

Here, $\mathfrak{M}(\mu):=\sup \left\{s \in\left[0, \operatorname{VaR}_{p}(X)\right]: \int_{\operatorname{VaR}_{p}(X)-s}^{\operatorname{VaR}(X)} \bar{F}(t) d t=\mu\right\}$.

Proof. For any $\nu \in\left[\nu(\mu), \operatorname{VaR}_{p}(X)+\mu-E X\right] \cap \mathfrak{N}_{\mu}$, we have $\pi(\tilde{R}[X ; \mu, \nu])=(1+\rho) \mu$ and

$$
C \operatorname{VaR}_{p}(\tilde{R}[X ; \mu, \nu])-\operatorname{VaR}_{p}(\tilde{R}[X ; \mu, \nu])=0
$$

Moreover, we get

$$
\operatorname{VaR}_{p}(\tilde{I}[X ; \mu, \nu])-E \tilde{I}[X ; \mu, \nu]=\operatorname{VaR}_{p}(X)+\mu-E X-\nu,
$$

which is non-negative and non-increasing in $\nu$. Therefore, $h_{2}(\mu, \nu)$ is non-increasing in $\nu$, which completes the proof.

Remark 4.1. It is necessary to determine whenever $\nu^{*}(\mu)=\mathfrak{M}(\mu)$. Let

$$
\varphi(\mu):=\int_{E X-\mu}^{V a R_{p}(X)} \bar{F}(t) d t-\mu, 0 \leq \mu \leq \mu_{0} .
$$


Clearly, $\varphi(\mu)$ is a decreasing function with $\varphi(0)=\int_{E X}^{V a R_{p}(X)} \bar{F}(t) d t \geq 0$ and

$$
\varphi\left(\mu_{0}\right)=\int_{E X-\mu_{0}}^{V a R_{p}(X)} \bar{F}_{X}(t) d t-\mu_{0} \leq E\left(X \wedge\left(\operatorname{VaR}_{p}(X)+\mu_{0}-E X\right)\right)-\mu_{0}=0 .
$$

Thus,

$$
\mu_{0}^{s}=: \inf \left\{0 \leq \mu \leq \mu_{0}: \varphi(\mu) \leq 0\right\}
$$

is well-defined, and in turn, $\nu^{*}(\mu)=\operatorname{VaR}_{p}(X)+\mu-E X$ if and only if $\mu \geq \mu_{0}^{s}$.

Now, one may find $\mu^{*}$ and solve minimisation problem (2.3), by assuming that $\mu$ falls into one of the following three intervals: $\left[0, \mu_{0}^{s}\right],\left[\mu_{0}^{s}, \mu_{0}\right]$ and $\left[\mu_{0},+\infty\right]$. As mentioned earlier, $\mu$ is upper-bounded by $\frac{P}{1+\rho}$, and therefore, the analysis is again divided according to the value of $P$.

2a) If $P /(1+\rho)<\mu_{0}^{s}$, we have $\mu \in[0, P /(1+\rho)]$ and $\mu<\mu_{0}^{s}$. Thus, $\nu^{*}(\mu)=\mathfrak{M}(\mu)$ and $C \operatorname{VaR}_{p}\left(\tilde{R}\left[X ; \mu, \nu^{*}(\mu)\right]\right)-\operatorname{VaR}_{p}\left(\tilde{R}\left[X ; \mu, \nu^{*}(\mu)\right]\right)=0$ are true according to Remarks 3.1 and 4.1. Consequently,

$$
h_{2}\left(\mu, \nu^{*}(\mu)\right)=g\left(a_{1}(P-(1+\rho) \mu), \lambda K(\mu)\right)+\left(\left(1+a_{2}\right)(1+\rho)-c\right) \mu+c E X+V a R_{p}(X)-\mathfrak{M}(\mu)
$$

where $K(\mu)=: \operatorname{VaR}_{p}(X)-E X+\mu-\mathfrak{M}(\mu)$. Clearly,

$$
K^{\prime}(\mu)=1-\mathfrak{M}^{\prime}(\mu)=1-\frac{1}{\bar{F}\left(\operatorname{VaR}_{p}(X)-\mathfrak{M}(\mu)\right)} \leq 0 .
$$

Thus, if $\left(1+a_{2}\right)(1+\rho)-c-\mathfrak{M}^{\prime}(\mu) \leq 0$ is satisfied for any $\mu \in[0, P /(1+\rho)]$, then the minimum value of $h_{2}\left(\mu, \nu^{*}(\mu)\right)$ is attained at $\hat{\mu}_{1}^{*}=\frac{P}{1+\rho}$. Otherwise, a numerical solution will be employed.

2b) If $\frac{P}{1+\rho} \in\left[\mu_{0}^{s}, \mu_{0}\right)$, we give separate discussions for $\mu \in\left[0, \mu_{0}^{s}\right]$ and $\mu \in\left[\mu_{0}^{s}, P /(1+\rho)\right]$.

For any $\mu \in\left[0, \mu_{0}^{s}\right]$, we use similar arguments as used in case $\left.2 \mathrm{a}\right)$. The minimum value of $h_{2}\left(\mu, \nu^{*}(\mu)\right)$ is attained at $\hat{\mu}_{2}^{*}=\mu_{0}^{s}$, if $\left(1+a_{2}\right)(1+\rho)-c-\mathfrak{M}^{\prime}(\mu) \leq 0$ is satisfied for any $\mu \in\left[0, \mu_{0}^{s}\right]$. Otherwise, a numerical solution may be employed.

For any $\mu \in\left[\mu_{0}^{s}, P /(1+\rho)\right]$, Remarks 3.1 and 4.1 yield that $\nu^{*}(\mu)=\operatorname{VaR}_{p}(X)+\mu-E X$ and

$$
C \operatorname{VaR}_{p}\left(\tilde{R}\left[X ; \mu, \nu^{*}(\mu)\right]\right)-\operatorname{VaR}_{p}\left(\tilde{R}\left[X ; \mu, \nu^{*}(\mu)\right]\right)=0
$$

which in turn imply $\operatorname{VaR}_{p}\left(\tilde{I}\left[X ; \mu, \nu^{*}(\mu)\right]\right)-E \tilde{I}\left[X ; \mu, \nu^{*}(\mu)\right]=0$. Thus,

$$
h_{2}\left(\mu, \nu^{*}(\mu)\right)=\left(\left(a_{2}-a_{1}\right)(1+\rho)+\rho-c\right) \mu+a_{1} P+(1+c) E X
$$

and therefore, its minimum is attained at $\hat{\mu}_{3}^{*}=\mu_{0}^{s}$ if $\left(a_{2}-a_{1}\right)(1+\rho)+\rho-c>0$, or $\hat{\mu}_{3}^{*}=\frac{P}{1+\rho}$, otherwise.

Finally, the minimum between $h_{2}\left(\hat{\mu}_{2}^{*}, \nu^{*}\left(\hat{\mu}_{2}^{*}\right)\right)$ and $h_{2}\left(\hat{\mu}_{3}^{*}, \nu^{*}\left(\hat{\mu}_{3}^{*}\right)\right)$ provides the global optimal solution to $(2.3)$.

2c) If $\frac{P}{1+\rho} \in\left[\mu_{0}, E X\right]$, our analysis is divided into three cases: $\mu \in\left[0, \mu_{0}^{s}\right], \mu \in\left[\mu_{0}^{s}, \mu_{0}\right]$ and $\mu \in\left[\mu_{0}, P /(1+\rho)\right]$.

The first subcase, $\mu \in\left[0, \mu_{0}^{s}\right]$, is similar to case $\left.2 \mathrm{a}\right)$. The minimum value of $h_{2}\left(\mu, \nu^{*}(\mu)\right)$ is attained at $\hat{\mu}_{4}^{*}=\mu_{0}^{s}$, if for any $\mu \in\left[0, \mu_{0}^{s}\right]$ we have $\left(1+a_{2}\right)(1+\rho)-c-\mathfrak{M}^{\prime}(\mu) \leq 0$. Otherwise, a numerical solution is needed.

Whenever $\mu \in\left[\mu_{0}^{s}, \mu_{0}\right]$, similar arguments used to derive $\hat{\mu}_{3}^{*}$ show that the minimum value of $h_{2}\left(\mu, \nu^{*}(\mu)\right)$ is attained at $\hat{\mu}_{5}^{*}=\mu_{0}^{s}$ if $\left(a_{2}-a_{1}\right)(1+\rho)+\rho-c>0$, or $\hat{\mu}_{5}^{*}=\mu_{0}$, otherwise. 
Finally, for any $\mu \in\left[\mu_{0}, \frac{P}{1+\rho}\right]$, Proposition 3.2 suggests that the local solution is found via the minimisation of $h_{2}\left(\mu, \operatorname{VaR}_{p}(X)+\mu-E X\right)$. Specifically, we have

$$
\operatorname{VaR}_{p}\left(\tilde{I}\left[X ; \mu, \operatorname{VaR}_{p}(X)+\mu-E X\right]\right)-E \tilde{I}\left[X ; \mu, \operatorname{VaR} R_{p}(X)+\mu-E X\right]=0,
$$

and hence

$$
h_{2}\left(\mu, \operatorname{VaR}_{p}(X)+\mu-E X\right)=a_{1} P+(1+c) E X+\left(\left(1-a_{1}\right) \rho-a_{1}-c\right) \mu+g\left(a_{2}(1+\rho) \mu, \vartheta \mathcal{H}(\mu)\right)
$$

where

$$
\mathcal{H}(\mu):=\frac{1}{1-p}\left(\mu-E\left(X \wedge\left(\operatorname{VaR}_{p}(X)+\mu-E X\right)\right)\right) .
$$

If $\left(1-a_{1}\right) \rho-a_{1} \geq c$ (which is expected to hold under a realistic parametrisation), then $\hat{\mu}_{6}^{*}=\mu_{0}$. Otherwise, a numerical solution is employed.

We now give a numerical illustration of the above derivations, and in fact we solve optimisation problems (2.1) and (2.3) under a realistic parametrisation as suggested in QIS5. Alternatively, a short description is provided in Asimit et al. (2015). We also compare our results with the optimal contract given by Proposition 4.2 of Chi and Tan (2013), where the RM's have not been included in the objective function.

Example 4.1. In this example, we consider two different distributions for $X$. The first distribution is assumed to be Pareto with shape parameter $\alpha=3$ and scale parameter $\theta=1,000$, and therefore, $\bar{F}(x)=(1,000 /(x+1,000))^{3}$. The second one is a Log-Normal distribution with location parameter $\mu_{L N}=5.786$ and scale parameter $\sigma_{L N}=0.926$. The parameter values are chosen such that the two distributions have the same value for $E X$ and $V a R_{0.8}(X)$ respectively. The choice of a confidence level of $80 \%$ is made, since anecdotically, this level represents a rough estimate of the minimal capital that an insurer needs to hold to keep its business running. We also choose $\lambda=6 \% / 1.04$, CoC $=6 \%, \sigma_{P R}=0.1$ and $\sigma_{R R}=0.11$ as suggested in QIS5 for Fire and Other Property Damage, while $d=1.56, n=1$ and $\Delta_{n}=3 \%$ are suggested in Asimit et al. (2015). We set RecR $=50 \%$, which is the benchmark value used in Solvency II, and $q=6.04 \%$ that represents the one-year default probability of a B-rating reinsurer calibrated in QIS5. Finally, it is also assumed that $\rho=0.5$ and $l=3$.

Assuming that the reinsurer adopts an expected value premium principle as defined in (4.1), the feasible region of $\mu$ is upper-bounded by $\frac{P}{1+\rho}$ as the premium charged by the reinsurer should not exceed the gross premium received by the insurer. Thus, we now turn to the discussion of choosing a proper value for $P$. As analysed in the derivation of closed-form solutions to the optimisation problems (2.1) and (2.3), the feasible region of $P$, i.e. $[0,(1+\rho) E X]$, is further divided into two subintervals, $(1 a)\left[0,(1+\rho) E\left(X \wedge \operatorname{VaR}_{p}(X)\right)\right]$ and $(1 b)\left[(1+\rho) E\left(X \wedge \operatorname{VaR}_{p}(X),(1+\rho) E X\right]\right.$, for problem (2.1), while in problem (2.3) three sub-intervals of feasible value of $P$ are considered, $(2 a)\left[0,(1+\rho) \mu_{0}^{s}\right]$, $(2 b)\left[(1+\rho) \mu_{0}^{s},(1+\rho) \mu_{0}\right]$ and $(2 c)\left[(1+\rho) \mu_{0},(1+\rho) E X\right]$. Thus, our numerical illustration is divided according to these five intervals of $P$. Although the numerical boundaries of these five intervals are different under the two distributions of $X$ considered in this example, we try our best to employ the same value of $P$ for both distributions when each interval is considered, and therefore our optimal solutions may be compared across the two distributions. In particular, Table 4.1 summarises the numerical intervals of $P$ 
under Pareto and Log-Normal distributions respectively, together with the chosen value of $P$ from each interval.

\begin{tabular}{|c|c|c|c|c|}
\hline Model & Interval of $P$ & Pareto & Log-Normal & $P$ \\
\hline \hline \multirow{3}{*}{$(2.1)$} & $(1 a)$ & {$[0,728.07]$} & {$[0,739.51]$} & 700 \\
\cline { 2 - 5 } & $(1 b)$ & {$[728.07,750]$} & {$[739.51,750]$} & 740 \\
\hline \multirow{3}{*}{$(2.3)$} & $(2 a)$ & {$[0,591.76]$} & {$[0,555.46]$} & 555 \\
\cline { 2 - 5 } & $(2 b)$ & {$[591.76,727.96]$} & {$[555.46,739.46]$} & 700 \\
\cline { 2 - 5 } & $(2 c)$ & {$[727.96,750]$} & {$[739.46,750]$} & 740 \\
\hline
\end{tabular}

TABLE 4.1. Parametrisation of $P$.

For each of the five cases described above, Figure 4.1 contains the plots of the optimal ceded risk when $X$ is Pareto and Log-Normal distributed. We find that, under the same optimisation model and parametrisation, the insurer always retains more risk by itself when $X$ is Log-Normal distributed as compared to the Pareto case. This is not surprising, since Pareto has a heavier tail than Log-Normal.

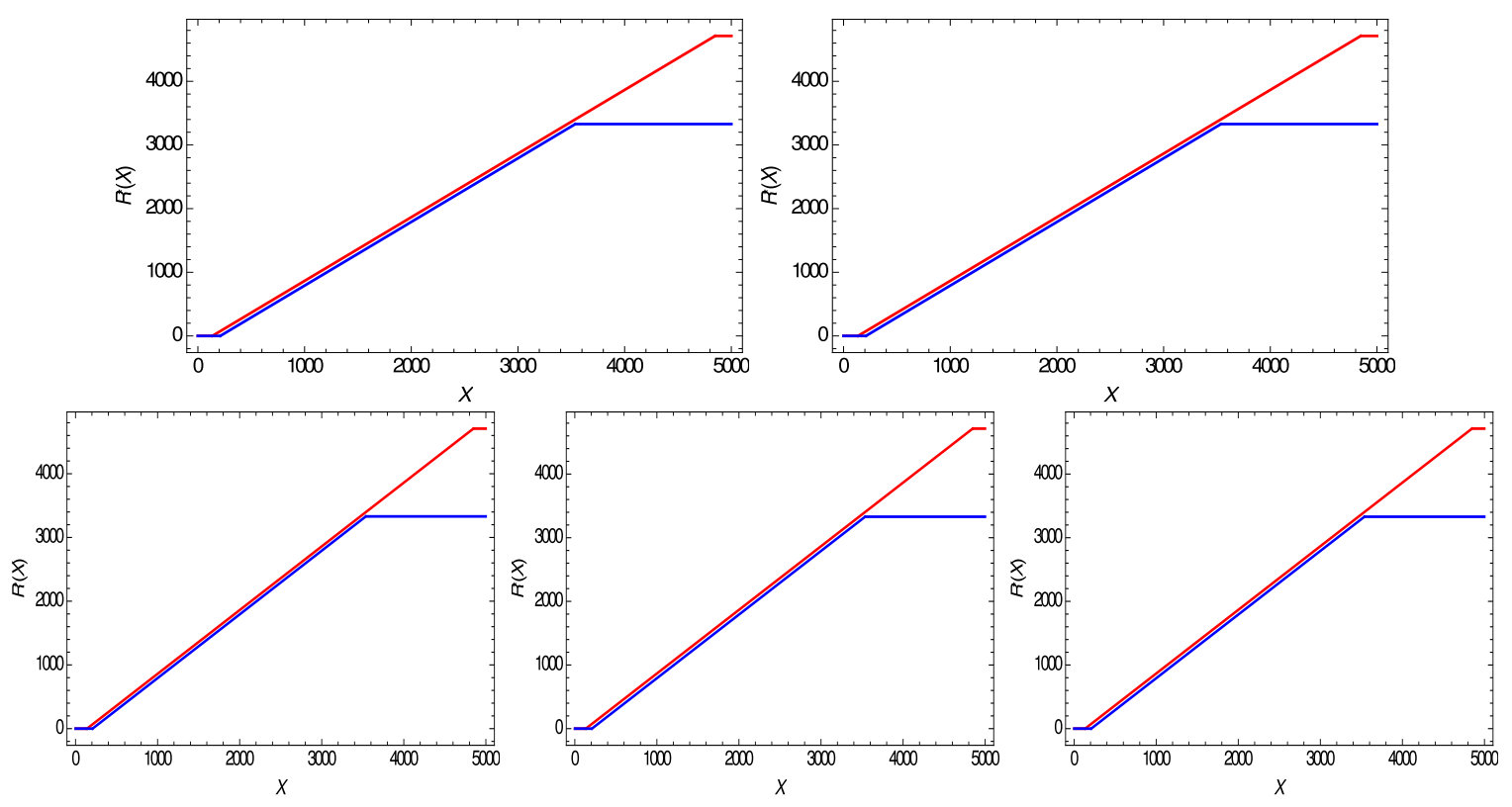

Figure 4.1. Closed-form solution under expected value principle with a Pareto distribution (red line) and a Log-Normal distribution (blue line), where the top row displays (1a) and (1b) and the bottom row shows (2a), (2b) and (2c).

Table 4.2 provides the solutions across the two optimisation models (2.1) and (2.3). In all of the ten examples investigated, the global optimal reinsurance contracts have the same two-layer form

$$
R^{*}[X]=\mathcal{L}_{\left(\operatorname{VaR}_{p}(X)-\nu^{*}, \operatorname{VaR}_{p}(X)\right]}(X)
$$

where the values of $\nu^{*}$ are summarised in Table 4.2 together with the corresponding expected value of the reinsurer's risk $\mu^{*}$. Also, under our parametrisation, $\operatorname{Va}_{p}(X)=4,848.04$ in the Pareto distribution 
case, while $\operatorname{VaR}_{p}(X)=3,537.65$ in the Log-Normal distribution setting. The conclusion drawn from Figure 4.1, that the insurer always retains more risk under Log-Normal distribution assumption than Pareto, is affirmed by studying Table 4.2. If we compare the values of $\nu^{*}$ and $\mu^{*}$ across optimisation models, we find that there is only negligible differences between the optimal contracts solved under the two methodologies where the RM is calculated either with or without applying the Log-Normal approximation. Thus, we can conclude that the optimal reinsurance contract is quite robust with respect to the two methodologies of the RM evaluation within Solvency II.

\begin{tabular}{|c|c|c|c|c|}
\hline Model & $P$ & Distribution & $\nu^{*}$ & $\mu^{*}$ \\
\hline \multirow{4}{*}{$(2.1)$} & \multirow{2}{*}{700} & Pareto & $4,712.81$ & 373.36 \\
\hline & & Log-Normal & $3,328.37$ & 309.84 \\
\hline & \multirow{2}{*}{740} & Pareto & $4,712.84$ & 373.38 \\
\hline & & Log-Normal & $3,328.38$ & 309.85 \\
\hline \multirow{6}{*}{$(2.3)$} & \multirow{2}{*}{555} & Pareto & $4,707.87$ & 370 \\
\hline & & Log-Normal & $3,330.21$ & 311.10 \\
\hline & \multirow{2}{*}{700} & Pareto & $4,713.97$ & 374.15 \\
\hline & & Log-Normal & $3,329.68$ & 310.74 \\
\hline & \multirow{2}{*}{740} & Pareto & $4,713.83$ & 374.06 \\
\hline & & Log-Normal & $3,329.58$ & 310.67 \\
\hline
\end{tabular}

TABLE 4.2. Numerical illustration of the closed-form solution under the expected value premium principle.

Finally, it would be interesting to compare our results with the optimal contract when the objective function does not take into account the change in the RM's, which can be found in Proposition 4.2 in Chi and Tan (2013), and it is

$$
R^{*}[X]=\mathcal{L}_{\left(\operatorname{VaR}_{\beta^{*}}(X), \operatorname{VaR}_{p}(X)\right]}(X) \text {, with } \beta^{*}=1 / 3 .
$$

It is found that $\operatorname{VaR}_{\beta^{*}}(X)=144.71$ in the Pareto distribution setting, while $\operatorname{VaR}_{\beta^{*}}(X)=218.58$ in the Log-Normal distribution case. Thus, the insurer always retains less risk if the RM's are included in the optimisation problem. In other words, our optimal contracts are more conservative than the one from Chi and Tan (2013).

4.2. Wang's premium principle. The expected value premium principle allows us to provide closedform solutions to our optimisation problems. Next, we numerically find the optimal contracts for a large family of premium principles, namely Wang's premium principle, for which closed-form solutions usually seem difficult to be found. There is a rich literature on insurance pricing that recognises the advantages of this pricing method (see for example, Wang, 2000, Wang and Young, 1998, Wang et al., 1997, Yaari, 1987). Under this premium principle, we have

$$
\pi(X):=(1+\rho) \int_{0}^{\infty} w(\bar{F}(x)) d x
$$


where the distortion function $w:[0,1] \mapsto[0,1]$ is a non-decreasing and concave function such that $w(0)=0$ and $w(1)=1$. The safety loading coefficient, $\rho$, is employed as in the expected value premium principle. Thus, (4.5) also includes the expected value principle as defined in (4.1), and therefore, allows us to conveniently compare our numerical results with the closed-form solutions. Jones and Zitikis (2003) investigates the empirical estimator for (4.5), which is:

$$
\hat{\pi}(X)=(1+\rho) \sum_{i=1}^{n} \phi_{i} x_{i, n},
$$

where $\phi_{i}=w\left(1-\frac{i-1}{n}\right)-w\left(1-\frac{i}{n}\right)$ and $x_{1, n} \leq x_{2, n} \leq \ldots \leq x_{n, n}$ are the sample ordered statistics.

Recall that we deal with the following family of admissible ceded loss functions:

$$
\begin{aligned}
& R\left[X ; d_{1}, d_{2}, \mu, \nu\right]:=\mathcal{L}_{\left(0, d_{1}\right]}(X)+\mathcal{L}_{\left(\operatorname{VaR}_{p}(X)+d_{1}-\nu, d_{2}\right]}(X) \\
& \text { s.t. } E R\left[X ; d_{1}, d_{2}, \mu, \nu\right]=\mu, 0 \leq d_{1} \leq \nu \leq \operatorname{VaR}_{p}(X) \leq d_{2} \leq x_{F}, \\
& \qquad \int_{V a R_{p}(X)-\nu}^{V a R_{p}(X)} \bar{F}(x) d x \leq \mu \leq E X-\int_{\nu}^{V a R_{p}(X)} \bar{F}(x) d x,
\end{aligned}
$$

which is discussed in the proof of Theorem 3.1. We further assume that the loss distribution is discrete in order to use the sample estimates for the reinsurance premium. This is not a restrictive assumption from the practical point of view, since this sample represents the claim history. If there is no reliable claim history but a proxy model is socially accepted, then one may draw a sample form this model. That is, we observe a sample of size $n$, which without loss of generality is assumed to be increasingly ordered, e.g. $x_{1} \leq x_{2} \leq \ldots \leq x_{n}$. Therefore, the reinsurance premium under Wang's premium principle becomes

$$
\pi\left(R\left[X ; d_{1}, d_{2}, \mu, \nu\right]\right)=(1+\rho) \sum_{i=1}^{n} \phi_{i} \times\left(x_{i} \wedge d_{1}+\left(x_{i}-x_{\lceil n p\rceil}-d_{1}+\nu\right)_{+}-\left(x_{i}-d_{2}\right)_{+}\right),
$$

where $x_{\lceil n p\rceil}$ is the sample estimation of $\operatorname{VaR}(X)$. The feasibility conditions for the optimisation problem from (2.1) are

$$
\pi\left(R\left[X ; d_{1}, d_{2}, \mu, \nu\right]\right) \leq P
$$

while for (2.3) they are

$$
\pi\left(R\left[X ; d_{1}, d_{2}, \mu, \nu\right]\right) \leq P, \nu \leq \operatorname{VaR}(X)+\mu-E X .
$$

Note that $\int_{V^{2} R_{p}(X)-\nu}^{V a R_{p}(X)} \bar{F}(x) d x$ and $\int_{\nu}^{V a R_{p}(X)} \bar{F}(x) d x$ can be replaced by

$$
E\left(X-\operatorname{VaR}_{p}(X)+\nu\right)_{+}-E\left(X-\operatorname{VaR}_{p}(X)\right)_{+}=\frac{1}{n} \sum_{i=1}^{n}\left(\left(x_{i}-x_{\lceil n p\rceil}+\nu\right)_{+}-\left(x_{i}-x_{\lceil n p\rceil}\right)_{+}\right),
$$

and

$$
E(X-\nu)_{+}-E\left(X-\operatorname{VaR}_{p}(X)\right)_{+}=\frac{1}{n} \sum_{i=1}^{n}\left(\left(x_{i}-\nu\right)_{+}-\left(x_{i}-x_{\lceil n p\rceil}\right)_{+}\right),
$$

respectively. Therefore, we deal with two non-convex optimisation problems that need to be transformed into implementable formulations in some commercial optimisation software. The next two propositions show how the optimisation problems (2.1) and (2.3) can be reformulated as Mixed Integer Nonlinear Programming $(M I N L P)$ with linear constraints that can be efficiently solved. The idea behind implementing (2.1) and (2.3) is the same, and thus, we only show Proposition 4.2. 
Proposition 4.2. Denote $\mathcal{A}=\{0,1\}$, and let $M$ be such that $M \geq 2 x_{n}$. Solving

$$
\min _{\mu, \nu, d_{1}, d_{2}} H_{1}\left(R\left[X ; d_{1}, d_{2}, \mu, \nu\right]\right) \quad \text { subject to (4.6), (4.7) and (4.9) }
$$

is equivalent to

$$
\begin{aligned}
& \min _{\left(d_{1}, d_{2}, \mu, \nu, \boldsymbol{u}, \boldsymbol{v}, \boldsymbol{w}\right) \in \Re \times \Re \times \Re \times \Re \times \Re^{n} \times \Re^{n} \times \Re^{n}}\left\{-\nu-c \mu+(1+\rho) \boldsymbol{\phi}^{T}(\boldsymbol{u}+\boldsymbol{v}-\boldsymbol{w})\right. \\
& (\boldsymbol{s}, \boldsymbol{t}, \boldsymbol{\eta}, \boldsymbol{\theta}, \boldsymbol{\gamma}, \boldsymbol{\xi}, \boldsymbol{\psi}) \in \Re^{n} \times \Re^{n} \times \mathcal{A}^{n} \times \mathcal{A}^{n} \times \mathcal{A}^{n} \times \mathcal{A}^{n} \times \mathcal{A}^{n} \\
& \left.+g\left(a_{2}(1+\rho) \boldsymbol{\phi}^{T}(\boldsymbol{u}+\boldsymbol{v}-\boldsymbol{w}), b_{2} \mu\right)+g\left(a_{1} P-a_{1}(1+\rho) \boldsymbol{\phi}^{T}(\boldsymbol{u}+\boldsymbol{v}-\boldsymbol{w}), b_{1} \bar{x}-b_{1} \mu\right)\right\} \\
& \text { s.t. } 0 \leq d_{1} \leq \nu \leq x_{\lceil n p\rceil} \leq d_{2} \leq x_{n} \\
& -\boldsymbol{x}+\boldsymbol{u} \leq \boldsymbol{0},-d_{1} 1+\boldsymbol{u} \leq \boldsymbol{0}, \\
& \boldsymbol{x}-\boldsymbol{u}-M \boldsymbol{\eta} \leq \boldsymbol{0},-M \boldsymbol{1}+d_{1} \boldsymbol{1}-\boldsymbol{u}+M \boldsymbol{\eta} \leq \boldsymbol{0}, \\
& \boldsymbol{x}-\left(x_{\lceil n p\rceil}+d_{1}-\nu\right) \boldsymbol{1}-\boldsymbol{v} \leq \boldsymbol{O},-\boldsymbol{v} \leq \boldsymbol{O}, \\
& -\boldsymbol{x}+\left(x_{\lceil n p\rceil}+d_{1}-\nu\right) \boldsymbol{1}+\boldsymbol{v}-\mathbf{M} \boldsymbol{\theta} \leq \boldsymbol{0},-M \boldsymbol{1}+\boldsymbol{v}+M \boldsymbol{\theta} \leq \boldsymbol{0}, \\
& \boldsymbol{x}-d_{2} \boldsymbol{1}-\boldsymbol{w} \leq \boldsymbol{0},-\boldsymbol{w} \leq \boldsymbol{O}, \\
& -\boldsymbol{x}+d_{2} \boldsymbol{1}+\boldsymbol{w}-M \gamma \leq \boldsymbol{0},-M \boldsymbol{1}+\boldsymbol{w}+M \gamma \leq \boldsymbol{0}, \\
& \boldsymbol{x}-\left(x_{\lceil n p\rceil}-\nu\right) \boldsymbol{1}-\boldsymbol{s} \leq \boldsymbol{0},-\boldsymbol{s} \leq \boldsymbol{0}, \\
& -\boldsymbol{x}+\left(x_{\lceil n p\rceil}-\nu\right) \boldsymbol{1}+s-M \boldsymbol{\xi} \leq \boldsymbol{0},-M 1+s+M \boldsymbol{\xi} \leq \boldsymbol{0}, \\
& \boldsymbol{x}-\nu 1-\boldsymbol{t} \leq \boldsymbol{0},-\boldsymbol{t} \leq \boldsymbol{0}, \\
& -x+\nu 1+t-M \psi \leq 0,-M 1+t+M \psi \leq 0, \\
& 1^{T} s-n K-n \mu \leq 0, \\
& n \mu-n \bar{x}+\boldsymbol{1}^{T} \boldsymbol{t}-n K \leq 0, \\
& -P+(1+\rho) \phi^{T}(\boldsymbol{u}+\boldsymbol{v}-\boldsymbol{w}) \leq 0, \\
& n \mu-\boldsymbol{1}^{T}(\boldsymbol{u}+\boldsymbol{v}-\boldsymbol{w})=0,
\end{aligned}
$$

where $\bar{x}$ represents the sample mean and $K=\frac{1}{n} \sum_{i=1}^{n}\left(x_{i}-x_{\lceil n p\rceil}\right)_{+}$.

Proof. The relevant terms of the objective function are

$$
\begin{aligned}
-\nu & -\frac{c}{n} \sum_{i=1}^{n}\left(x_{i} \wedge d_{1}+\left(x_{i}-\left(x_{\lceil n p\rceil}+d_{1}-\nu\right)\right)_{+}-\left(x_{i}-d_{2}\right)_{+}\right) \\
& +(1+\rho) \sum_{i=1}^{n} \phi_{i}\left(x_{i} \wedge d_{1}+\left(x_{i}-\left(x_{\lceil n p\rceil}+d_{1}-\nu\right)\right)_{+}-\left(x_{i}-d_{2}\right)_{+}\right) \\
& +g\left(a_{1} P-a_{1}(1+\rho) \sum_{i=1}^{n} \phi_{i}\left(x_{i} \wedge d_{1}+\left(x_{i}-\left(x_{\lceil n p\rceil}+d_{1}-\nu\right)\right)_{+}-\left(x_{i}-d_{2}\right)_{+}\right), b_{1} \bar{x}-b_{1} \mu\right) \\
& +g\left(a_{2}(1+\rho) \sum_{i=1}^{n} \phi_{i}\left(x_{i} \wedge d_{1}+\left(x_{i}-\left(x_{\lceil n p\rceil}+d_{1}-\nu\right)\right)_{+}-\left(x_{i}-d_{2}\right)_{+}\right), b_{2} \mu\right),
\end{aligned}
$$


by taking into account (4.8) and the fact that $x_{1} \leq x_{2} \leq \ldots \leq x_{n}$. Now, note that

$$
x \wedge y=z \Longleftrightarrow z \leq x, z \leq y, x-M \eta \leq z, y-M(1-\eta) \leq z
$$

and

$$
\max \{x, y\}=z \Longleftrightarrow z \geq x, z \geq y, x+M \eta \geq z, y+M(1-\eta) \geq z
$$

where $M$ is a large number and $\eta$ is a binary variable, i.e. $\eta \in \mathcal{A}$. By multiple use of the latter in (4.11) one may conclude the first reformulation, where any $M \geq 2 x_{n}$ satisfies all required inequality constraints. This completes the proof.

The next proposition gives the reformulation of optimisation problem (2.3) and is based on the objective function without Log-Normal approximation for the RM's. Before stating the next proposition, we note that (2.2) and (4.6) yield that

$$
\begin{aligned}
C \operatorname{VaR}_{p}\left(R\left[X ; \mu, \nu, d_{1}, d_{2}\right]\right)-\operatorname{VaR}_{p}\left(R\left[X ; \mu, \nu, d_{1}, d_{2}\right]\right) & =\frac{1}{1-p} \int_{V a R_{p}(X)}^{d_{2}} \bar{F}(x) d x \\
& =\frac{1}{1-p}\left(E\left(X-\operatorname{VaR}_{p}(X)\right)_{+}-E\left(X-d_{2}\right)_{+}\right) \\
& =\frac{1}{(1-p) n} \sum_{i=1}^{n}\left(\left(x_{i}-x_{\lceil n p\rceil}\right)_{+}-\left(x_{i}-d_{2}\right)_{+}\right) .
\end{aligned}
$$

Proposition 4.3. Solving

$$
\min _{\mu, \nu, d_{1}, d_{2}} H_{2}\left(R\left[X ; d_{1}, d_{2}, \mu, \nu\right]\right) \quad \text { subject to (4.6), (4.7) and (4.10) }
$$


is equivalent to

$$
\begin{aligned}
& \min _{\left(d_{1}, d_{2}, \mu, \nu, \boldsymbol{u}, \boldsymbol{v}, \boldsymbol{w}\right) \in \Re \times \Re \times \Re \times \Re \times \Re^{n} \times \Re^{n} \times \Re^{n}}\left\{-\nu-c \mu+(1+\rho) \boldsymbol{\phi}^{T}(\boldsymbol{u}+\boldsymbol{v}-\boldsymbol{w})\right. \\
& (\boldsymbol{s}, \boldsymbol{t}, \boldsymbol{\eta}, \boldsymbol{\theta}, \boldsymbol{\gamma}, \boldsymbol{\xi}, \boldsymbol{\psi}) \in \Re^{n} \times \Re^{n} \times \mathcal{A}^{n} \times \mathcal{A}^{n} \times \mathcal{A}^{n} \times \mathcal{A}^{n} \times \mathcal{A}^{n} \\
& +g\left(a_{2}(1+\rho) \boldsymbol{\phi}^{T}(\boldsymbol{u}+\boldsymbol{v}-\boldsymbol{w}), \frac{\vartheta}{(1-p) n}\left(n K-\boldsymbol{1}^{T} \boldsymbol{w}\right)\right) \\
& \left.+g\left(a_{1} P-a_{1}(1+\rho) \boldsymbol{\phi}^{T}(\boldsymbol{u}+\boldsymbol{v}-\boldsymbol{w}), \lambda\left(x_{\lceil n p\rceil}-\nu\right)-\lambda(\bar{x}-\mu)\right)\right\} \\
& \text { s.t. } 0 \leq d_{1} \leq \nu \leq x_{\lceil n p\rceil} \leq d_{2} \leq x_{n} \\
& -\boldsymbol{x}+\boldsymbol{u} \leq \boldsymbol{0},-d_{1} 1+\boldsymbol{u} \leq \boldsymbol{0}, \\
& \boldsymbol{x}-\boldsymbol{u}-M \boldsymbol{\eta} \leq \boldsymbol{0},-M \boldsymbol{1}+d_{1} \boldsymbol{1}-\boldsymbol{u}+M \boldsymbol{\eta} \leq \boldsymbol{0}, \\
& \boldsymbol{x}-\left(x_{\lceil n p\rceil}+d_{1}-\nu\right) \boldsymbol{1}-\boldsymbol{v} \leq \boldsymbol{0},-\boldsymbol{v} \leq \boldsymbol{0}, \\
& -\boldsymbol{x}+\left(x_{\lceil n p\rceil}+d_{1}-\nu\right) \boldsymbol{1}+\boldsymbol{v}-\boldsymbol{M} \boldsymbol{\theta} \leq \boldsymbol{0},-M \boldsymbol{1}+\boldsymbol{v}+M \boldsymbol{\theta} \leq \boldsymbol{0}, \\
& \boldsymbol{x}-d_{2} \boldsymbol{1}-\boldsymbol{w} \leq \boldsymbol{0},-\boldsymbol{w} \leq \boldsymbol{O}, \\
& -\boldsymbol{x}+d_{2} \boldsymbol{1}+\boldsymbol{w}-M \gamma \leq \boldsymbol{0},-M 1+\boldsymbol{w}+M \gamma \leq \boldsymbol{0}, \\
& \boldsymbol{x}-\left(x_{\lceil n p\rceil}-\nu\right) \boldsymbol{1}-\boldsymbol{s} \leq \boldsymbol{0},-\boldsymbol{s} \leq \boldsymbol{0}, \\
& -\boldsymbol{x}+\left(x_{\lceil n p\rceil}-\nu\right) \boldsymbol{1}+s-M \boldsymbol{\xi} \leq \boldsymbol{0},-M \boldsymbol{1}+s+M \boldsymbol{\xi} \leq \boldsymbol{0}, \\
& \boldsymbol{x}-\nu 1-t \leq 0,-t \leq 0, \\
& -x+\nu 1+t-M \psi \leq 0,-M 1+t+M \psi \leq 0, \\
& \mathbf{1}^{T} s-n K-n \mu \leq 0 \\
& n \mu-n \bar{x}+\boldsymbol{1}^{T} \boldsymbol{t}-n K \leq 0, \\
& \nu-\mu+\bar{x}-x_{\lceil n p\rceil} \leq 0, \\
& -P+(1+\rho) \phi^{T}(\boldsymbol{u}+\boldsymbol{v}-\boldsymbol{w}) \leq 0, \\
& n \mu-\boldsymbol{1}^{T}(\boldsymbol{u}+\boldsymbol{v}-\boldsymbol{w})=0 .
\end{aligned}
$$

With the help of Propositions 4.2 and 4.3, numerical solutions can be obtained for a large class of premium principles. In fact, we produce a discrete approximation of the objective functions that can be solved in any commercial optimisation software such as MATLAB, if the underlying distribution of $X$ is known. In particular, we illustrate further results for the expected value principle in Example 4.2, while results for the Wang's premium principle are presented in Example 4.3

Example 4.2. In this example, we provide numerical approximations of optimal solutions under the expected value premium principle. One set of 100 samples of size 1,000 is simulated from each of the two parametric models used in Example 4.1, so that the numerical results given in the two examples can be compared. As it has been seen from Example 4.1, optimal contracts found in all of the ten examples have the same two-layer form regardless of the value of $P$ chosen. Thus, we only provide examples for cases (1b) and (2c) with $P=740$, since these cases provide a large set of feasible solutions. 


\begin{tabular}{|c||c|c|}
\hline Loss Distribution & Model (2.1) & Model (2.3) \\
\hline \hline Pareto & $4,597.5$ & $4,596.9$ \\
\hline Log-Normal & $3,461.6$ & $3,462.3$ \\
\hline
\end{tabular}

TABLE 4.3. Empirical solutions of $\nu^{*}$ to optimisation models (2.1) and (2.3) under expected value principle with $P=740$.

Numerous examples have been employed and showed that the estimated value of $d_{2}^{*}$ has negligible deviation from its closed-form solution value $\operatorname{VaR}_{p}(X)$. However, variations exist in the estimated value of $d_{1}^{*}$, which is due to the lack of efficiency in using the MATLAB algorithm to solve MINLP. Nevertheless, we have seen cost-inefficiency in transferring very small liabilities when studying Example 4.1, and therefore, it is expected that the bottom-layer of the optimal contract to be strictly positive, i.e. $d_{1}^{*}=0$. Thus, in order to speed up the computation process, we can explicitly set $d_{1}^{*}=0$ and $d_{2}^{*}=x_{\lceil n p\rceil}$ and only compute $\nu^{*}$. Clearly, the optimal reinsurance contract is as given in equation (4.4), and the mean value of estimated $\nu^{*}$ is summarised in Table 4.3. The results are satisfactorily close to its corresponding closed-form value, since the relative errors are less than $2.5 \%$ in the Pareto distribution case and are less than $4 \%$ in the Log-Normal setting. Thus, it can be concluded that the numerical procedure is stable.

Example 4.3. The final example provides numerical illustrations to optimal reinsurance contracts under Wang's premium principle. In particular, we choose the Proportional Hazards Transform (PHT) principle, where $w(t)=t^{0.95}$. In addition, $\rho=0.38$ is set, while all other parameters remain the same as in Example 4.1. The value of $\rho$ is chosen in such manner that, under the PHT principle, the optimal contracts found in Example 4.1 are as expensive as priced under the expected value principle. Instead of simulating new samples, we use the same simulated data as in Example 4.2. Again, we obtain robust estimates for $d_{2}^{*}=V a R_{p}(X)$, while variation exists in the estimates of $d_{1}^{*}$. However, by a similar argument given in Example 4.2, it is unnatural for the insurer to cede very low layers from the total loss. Thus, it can be explicitly set $d_{1}^{*}=0$ and $d_{2}^{*}=\operatorname{Va} R_{p}(X)$. The optimal reinsurance contract has the form as in

\begin{tabular}{|c||c|c|}
\hline Loss Distribution & Model (2.1) & Model (2.3) \\
\hline \hline Pareto & $4,598.3$ & $4,598.3$ \\
\hline Log-Normal & $3,462.4$ & $3,460.9$ \\
\hline
\end{tabular}

TABLE 4.4. Empirical solutions of $\nu^{*}$ to optimisation models (2.1) and (2.3) under PHT premium principle with $P=740$.

equation (4.4), and a summary of the estimates of $\nu^{*}$ based on our 100 samples are given in Table 4.4. It can be seen that the optimal contracts found under PHT principle are very close to those found under the expected value principle, which can be explained by our choice for $\rho$.

\section{ACKnowledgments}

Chi's research was supported by a grant from the National Natural Science Foundation of China (No. 11471345) and a MOE (China) Project of Key Research Institute of Humanities and Social Sciences 
at Universities (13JJD790040). Junlei Hu would like to thank Cass Business School for funding her research.

\section{REFERENCES}

Acerbi, C. and Tasche, D. 2002. "On the Coherence of Expected Shortfall," Journal of Banking EG Finance, 26(7), 1487-1503.

Arrow, K.J. 1963. "Uncertainty and the Welfare Economics of Medical Care," American Economic Review, 53(5), 941-973.

Asimit, A.V., Badescu, A.M. and Cheung, K.C. 2013. "Optimal Reinsurance in the Presence of Counterparty Default Risk", Insurance: Mathematics and Economics, 53(3), 690-697.

Asimit, A.V., Badescu, A.M., Haberman, S. and Kim, E.-S. 2015. "Efficient Risk Allocation within a Non-life Insurance Group under Solvency II Regime", Insurance: Mathematics and Economics, Forthcoming.

Bellini, F. and Rosazza Gianin, E. 2012. "Haezendonck-Goovaerts Risk Measures and Orlicz Quantiles," Insurance: Mathematics and Economics, 51(1), 107-114.

Bernard, C. and Ludkovski, M. 2012. "Impact of Counterparty Risk on the Reinsurance Market," North American Actuarial Journal, 16(1), 87-111.

Biffis, E. and Millossovich, P. 2012. "Optimal Insurance with Counterparty Default Risk," Working Paper. Available at http://ssrn. com/abstract $=1634883$

Borch, K., 1960. "An Attempt to Determine the Optimum Amount of Stop Loss Reinsurance," Transactions of the 16th International Congress of Actuaries, vol. I, 597-610, Georges Thone, Brussels, Belgium.

Cai, J. and Tan, K.S. 2007. "Optimal Retention for a Stop-loss Reinsurance under the VaR and CTE Risk Measures," Astin Bulletin, 37 (1), 93-112.

Cai, J., Tan, K.S., Weng, C. and Zhang, Y. 2008. "Optimal Reinsurance under VaR and CTE Risk Measures," Insurance: Mathematics and Economics, 43(1), 185-196.

Cai, J. and Weng, C. 2015. "Optimal Reinsurance with Expectile", Scandinavian Actuarial Journal, Forthcoming.

Centeno, M.L. and Guerra, M. 2010. "The Optimal Reinsurance Strategy - The Individual Claim Case," Insurance: Mathematics and Economics, 46(3), 450-460.

Cheung, K.C. 2010. "Optimal Reinsurance Revisited - A Geometric Approach ," Astin Bulletin, 40(1), $221-239$.

Chi, Y. 2012. "Reinsurance Arrangements Minimizing the Risk-adjusted Value of An Insurer's Liability," Astin Bulletin, 42(2), 529-557.

Chi, Y. and Meng, H. 2014. "Optimal Reinsurance Arrangements in the Presence of Two Reinsurers," Scandinavian Actuarial Journal, Issue 5, 424-438.

Chi, Y. and Tan, K.S. 2011. "Optimal Reinsurance under VaR and CVaR Risk Measures: A Simplified Approach," Astin Bulletin, 41(2), 487-509.

Chi, Y. and Tan, K.S. 2013. "Optimal Reinsurance with General Premium Principles," Insurance: Mathematics and Economics, 52(2), 180-189.

European Commission 2009. "Directive 2009/138/EC of the European Parliament and of the Council of 25 November 2009 on the Taking-up and Pursuit of the Business of Insurance and Reinsurance (Solvency II)", Official Journal of the European Union L335.

European Commission 2010. "QIS5 Technical Specifications", available at http://ec.europa.eu/ internal_market/insurance/docs/solvency/qis5/201007/technical_specifications_en.pdf.

Goovaerts, M.J., Kaas, R., Dhaene, J. and Tang, Q. 2004. "Some New Classes of Consistent Risk Measures," Insurance: Mathematics and Economics, 34(3), 505-516. 
Goovaerts, M.J., Linders, D., Van Weert, K. and Tank, F. 2012. "On the Interplay Between Distortion, Mean Value and Haezendonck-Goovaerts Risk Measures," Insurance: Mathematics and Economics, 51(1), 10-18.

Guerra, M. and Centeno, M.L. 2008. "Optimal Reinsurance Policy: The Adjustment Coefficient and the Expected Utility Criteria," Insurance: Mathematics and Economics, 42(2), 529-539.

Guerra, M. and Centeno, M.L. 2010. "Optimal Reinsurance for Variance Related Premium Calculation Principles," Astin Bulletin, 40(1), 97-121.

Haezendonck, J. and Goovaerts, M.J. 1982. "A New Premium Calculation Principle Based on Orlicz Norms," Insurance: Mathematics and Economics, 1(1), 41-53.

Jones, B.L. and Zitikis, R. 2003. "Empirical Estimation of Risk Measures and Related Quantities", North American Actuarial Journal, 7(4), 44-54.

Kaluszka, M., 2001. "Optimal Reinsurance under Mean-variance Premium Principles," Insurance: Mathematics and Economics, 28(1), 61-67.

Kaluszka, M., 2005. "Truncated Stop Loss as Optimal Reinsurance Agreement in One-period Models," Astin Bulletin, 35(2), 337-349.

Kaluszka, M. and Okolewski, A. 2008. "An Extension of Arrow's Result on Optimal Reinsurance Contract," The Journal of Risk and Insurance, 75(2), 275-288.

Lu, Z., Liu, L., Shen, Q. and Meng, L. 2014. "Optimal Reinsurance Under VaR and CTE Risk Measures When Ceded Loss Function is Concave," Communications in Statistics - Theory and Methods, 43(15), 3223-3247.

Ludkovski, M. and Young, V.R. 2009. "Optimal Risk Sharing under Distorted Probabilities," Mathematics and Financial Economics, 2(2), 87-105.

Ohlin, J. 1969. "On a Class of Measures of Dispersion with Application to Optimal Reinsurance," Astin Bulletin, 5(2), 249-266.

Tan, K. S. and Weng, C. 2014. "Empirical Approach for Optimal Reinsurance Design," North American Actuarial Journal, 18(2), 315-342.

Van Heerwaarden, A.E., Kaas, R. and Goovaerts, M.J. 1989. "Optimal Reinsurance in Relation to Ordering of Risks," Insurance: Mathematics and Economics, 8(1), 11-17.

Verlaak, R. and Beirlant, J. 2003. "Optimal Reinsurance Programs: An Optimal Combination of Several Reinsurance Protections on a Heterogeneous Insurance Portfolio," Insurance: Mathematics and Economics, 33(2), 381-403.

Wang, S.S. 2000. "A Class of Distortion Operators for Pricing Financial and Insurance Risks," Journal of Risk and Insurance, 67(1), 15-36.

Wang, S.S. and Young, V.R. 1998. "Risk-adjusted Credibility Premiums Using Distorted Probabilities," Scandinavian Actuarial Journal, Issue 2, 143-165.

Wang, S.S., Young, V.R. and Panjer, H.H. 1997. "Axiomatic Characterization of Insurance Prices," Insurance: Mathematics and Economics, 21(2), 173-183.

Yaari, M.E. (1987). "The Dual Theory of Choice under Risk," Econometrica, 55(1), 95-115.

Young, V.R., 1999. "Optimal Insurance under Wang's Premium Principle," Insurance: Mathematics and Economics, 25(2), 109-122. 